\title{
Ratchet driven by quasimonochromatic noise
}

\author{
M. Arrayás, ${ }^{1}$ R. Mannella,,${ }^{1,2}$ P. V. E. McClintock, ${ }^{1}$ A. J. McKane, ${ }^{3}$ and N. D. Stein ${ }^{1}$ \\ ${ }^{1}$ Department of Physics, Lancaster University, Lancaster LA1 4YB, United Kingdom \\ ${ }^{2}$ Dipartimento di Fisica, Università di Pisa and INFM UdR Pisa, Piazza Torricelli 2, 56100 Pisa, Italy \\ ${ }^{3}$ Department of Theoretical Physics, University of Manchester, Manchester M13 9PL, United Kingdom
}

(Received 23 August 1999)

\begin{abstract}
The currents generated by noise-induced activation processes in a periodic potential are investigated analytically, by digital simulation and by performing analog experiments. The noise is taken to be quasimonochromatic and the potential to be a smoothed sawtooth. Two analytic approaches are studied. The first involves a perturbative expansion in inverse powers of the frequency characterizing quasimonochromatic noise and the second is a direct numerical integration of the deterministic differential equations obtained in the limit of weak noise. These results, together with the digital and analog experiments, show that the system does indeed give rise, in general, to a net transport of particles. All techniques also show that a current reversal exists for a particular value of the noise parameters.
\end{abstract}

PACS number(s): 05.40.Ca, 05.10.Gg, 02.50.Ey

\section{INTRODUCTION}

The nature of microscopic engines, such as molecular motors, has been the subject of much research over the last five or six years. This recent activity was stimulated by the possibility of noise-induced currents [1-4], and was motivated to a large extent by the desire to model protein motors. These are proteins which are connected to a biopolymer and catalyze the conversion of adenosine triphosphate (ATP) to adenosine diphosphate (ADP). The energy released by this process is used by the motor protein to generate motion along the biopolymer in one particular direction. This is modelled as a microscopic object moving unidirectionally along a onedimensional periodic structure [5]. It is this problem of rectifying processes at small scales that has stimulated most of the theoretical work in this area. A key ingredient is the presence of random Brownian forces. As a consequence it is natural to describe these stochastic ratchets, as they are frequently called, as a particle moving in a periodic potential subject to noise and to formulate this mathematically as a Langevin equation

$$
m \ddot{x}+\alpha \dot{x}+\partial_{x} V(x, t)=\xi(t)
$$

where $x(t)$ is the coordinate of the particle, $\alpha$ is a friction constant, $V(x, t)$ is a periodic asymmetric potential and $\xi(t)$ is the noise.

Before discussing Eq. (1) in more detail, we should point out that there are at least three other reasons for the renewed interest in such systems. The first is a new generation of experiments that can be performed in vitro [5], which has led to the increased sophistication of the models now studied. The second is the application of these ideas to nonbiological situations at small scales-the realm of nanotechnology. Finally, this problem throws up fundamental questions concerning irreversibility and the second law of thermodynamics. These issues are discussed extensively elsewhere [5], so we will just make the essential point that it is only if detailed balance holds that we can use considerations based on the second law to decide that no coherent unidirectional motion is possible [6].

The classification of different types of ratchets is in fact most easily carried out with reference to Eq. (1), since the terminology used to describe ratchets (correlation, flashing) is not always applied consistently. Nearly all studies neglect the inertial term in Eq. (1) and scale time by $\alpha$, so that the coefficient of the $\dot{x}$ term is unity. Most studies have focussed on the cases (i) $V(x, t)=V(x)$ is deterministic and $\xi(t)$ is nonwhite-noise (so that detailed balance does not hold), (ii) $V(x, t)=V(x) \phi(t)$ where $\phi(t)$ may be deterministic or random [7] and $\xi(t)$ is white noise. Since the main prerequisite for any ratchet is that the system does not obey detailed balance and, since detailed balance may be violated in many different ways, it is clear that many other forms are possible.

In this paper we will study a ratchet of the type (i) discussed above, sometimes called a correlation ratchet. In Sec. II the model is described in more detail: the potential is specified as is the type of noise-quasimonochromatic noise (QMN) [8], whose application to ratchets was first discussed in Ref. [2]. We study the model using analytic techniques in Secs. III and IV and by the digital and analog simulations which are discussed in Sec. V. We end with an analysis of results and conclusions. Some of our preliminary work has already been reported [9].

\section{MODEL}

In this section we will write down an explicit representation for a correlation ratchet acted upon by quasimonochromatic noise. Having said this, it only remains to specify the potential $V(x)$. We have already indicated that this function should be periodic and asymmetric. A natural choice would therefore be a sawtooth potential: as shown in Ref. [2], a sawtooth potential is the one which maximizes the current. However, in order to carry out analog experiments we take the first few Fourier modes of such a potential leading to the form 
$V(x)$

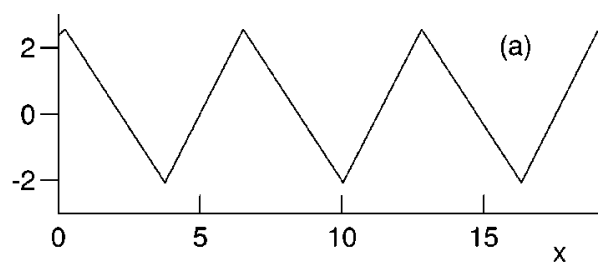

$V(x)$

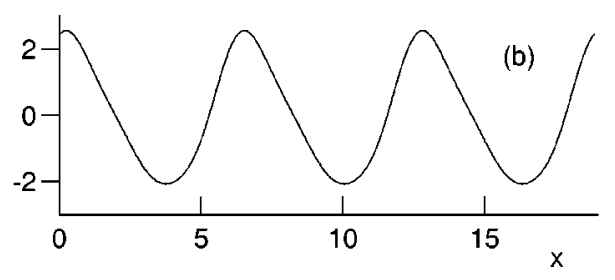

FIG. 1. (a) Sawtooth potential. (b) "Smoothed sawtooth" potential.

$$
V(x)=2 \cos x+\sin x+\frac{1}{3} \cos (2 x)+\frac{1}{10} \cos (3 x) .
$$

The sawtooth and the approximation to it, Eq. (2), are both shown in Fig. 1, where we can see that we are modelling the sawtooth potential by rounding the corners. This not only makes it easier to reproduce in an analog experiment, but it also removes possible singularities in the theoretical treatment: the "sharp corners" at the top and bottom of the potential would mean that the force that the particle would feel would be not well defined.

Therefore the model is defined by the Langevin equation

$$
\dot{x}+V^{\prime}(x)=\xi(t)
$$

where $V(x)$ is given by Eq. (2) and the noise $\xi(t)$ is taken to be Gaussian with zero mean and correlation function

$$
\left\langle\xi(\omega) \xi\left(\omega^{\prime}\right)\right\rangle=2 D 2 \pi C(\omega) \delta\left(\omega+\omega^{\prime}\right) .
$$

We choose $\xi$ to be quasimonochromatic noise (QMN) since it exactly suits our purposes: the noise cannot be white, for reasons described in the last section and it has the physically appealing feature of having a peak at a nonzero frequency in its power spectrum (hence the name [8] QMN), while being simple enough to allow analytic progress to be made. Specifically, the noise is defined by

$$
C^{-1}(\omega)=\left(\omega^{2}-\omega_{0}^{2}\right)^{2}+4 \Gamma^{2} \omega^{2}
$$

$C(\omega)$ is sharply peaked at the frequency $\left(\omega_{0}^{2}-2 \Gamma^{2}\right)^{1 / 2} \approx \omega_{0}$ in the limit $\Gamma \ll \omega_{0}$, and so we will frequently be working in this regime. This type of noise can also be viewed as the result of passing white noise through a harmonic oscillator filter:

$$
\ddot{\xi}+2 \Gamma \dot{\xi}+\omega_{0}^{2} \xi=\eta
$$

(hence the name "harmonic" [10,11], which is also often used) where the white noise $\eta$ has strength $D$.

We shall be mounting a three-pronged attack on the problem posed above: an analytic treatment based a small $D$ approximation, direct digital simulation of the Langevin equation, to be discussed in Sec. V, and an analog simulation also

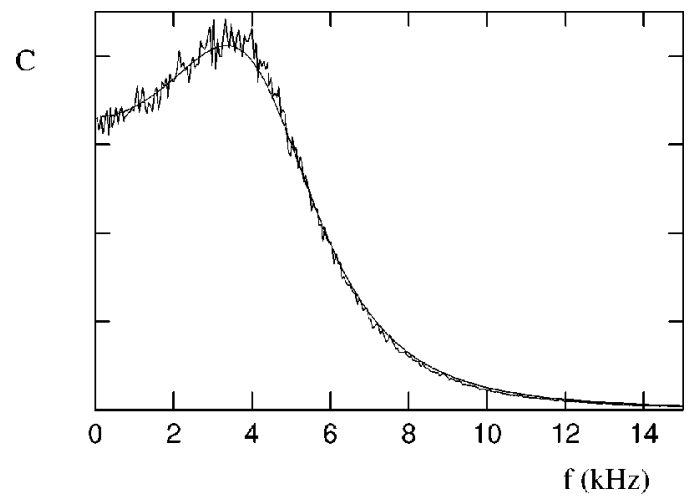

FIG. 2. Noise power spectrum for $\omega_{0}=31.6, \Gamma=16.7$. The jagged line is from experiment, and the smooth one from theory. The frequency $f=\omega / 2 \pi$.

to be discussed in Sec. V. In the case of the analog simulation, the first task, before simulating the Langevin equation itself, is to check the quality of the QMN produced by the analogue circuit. In order to accomplish this, we examine the QMN spectrum simulated by (6). Two examples are shown in Figs. 2 and 3.

It is evident that there is good agreement between the noise generated in the simulations and the theoretical result given by Eq. (5). It is also clear from these figures that the shape of the spectrum changes considerably depending on the values of the parameters $\Gamma$ and $\omega$. In fact, these figures illustrate the two regimes for QMN noise. The first (Fig. 2) is an example of the case $\omega_{0}^{2}>2 \Gamma^{2}$. The spectrum has a local minimum at $\omega=0$, rising to a maximum value at $\omega^{2}=\omega_{0}^{2}$ $-2 \Gamma^{2}$, and then falling off to zero as $\omega \rightarrow \infty$. As we have remarked already, in the limit $\omega_{0}^{2} \gg 2 \Gamma^{2}$ the peak becomes narrower and better defined. Moreover, for values of $\omega$ such that $\omega \ll \omega_{0}$, the spectrum is essentially flat and approximates well white noise. On the other hand Fig. 3 illustrates the case $\omega_{0}^{2}<2 \Gamma^{2}$, where the spectrum has a local maximum at $\omega$ $=0$ and falls away to zero as $\omega \rightarrow \infty$. So, in summary, if the damping parameter $\Gamma$ is small enough, the power spectrum has a peak at nonzero frequency. As $\Gamma$ increases, the peak broadens and moves towards zero frequency. For $\Gamma$ greater than a critical value of $\omega_{0} / \sqrt{2}$ the maximum of the power spectrum is at zero frequency. Our aim is to see how the current changes as the noise parameters $\Gamma$ and $\omega_{0}$ vary.

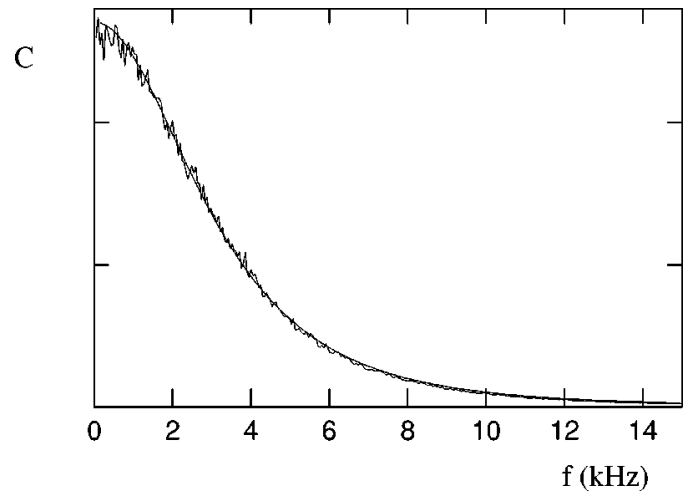

FIG. 3. Noise power spectrum for $\omega_{0}=31.6, \Gamma=33.3$. The jagged line is from experiment, and the smooth one from theory. The frequency $f=\omega / 2 \pi$. 


\section{GENERAL FORMALISM}

In this section we discuss the approach we will use to explore analytically the generation of noise-induced currents in the correlation ratchet introduced in the previous section. The method will involve an asymptotic analysis in the limit where the noise strength $D$ tends to zero. To construct the asymptotic expansion it is first necessary to formulate the problem defined by the Langevin equation (3) in a different form. There are at least two different ways to proceed. One is to write down an equivalent Fokker-Planck equation. Since the noise $\xi(t)$ in Eq. (3) is not white, it is first necessary to convert the process into an equivalent Markovian one with three degrees of freedom, $(x, \xi, \dot{\xi})$, say. Thus the FokkerPlanck equation will have the form of time-dependent partial differential equation in three dimensions. We shall not pursue this method here, instead we will use the approach of expressing the conditional probability $\langle\delta(x-x(t))\rangle_{\mathrm{IC}}$ as an average over all possible paths (or realizations of the process) with given initial conditions, denoted here by IC. These initial conditions specify not only the initial values of $x(t)$, but also of $\xi(t)$ and of $\dot{\xi}(t)$ at $t=t_{0}$. The explicit form for the path-integral is [12]

$$
P\left(x, t \mid \mathrm{IC}, t_{0}\right)=\langle\delta(x-x(t))\rangle_{\mathrm{IC}}=\int_{\mathrm{IC}} \mathcal{D} x \mathcal{P}[x] \delta(x-x(t)),
$$

where $\mathcal{D} x$ is the appropriate measure defined so that $P$ is correctly normalized and

$$
\mathcal{P}[x]=J[x] \exp -S[x] / D .
$$

Here $S[x]$ is the action functional, which will be discussed in more detail below, and $J[x]$ is the Jacobian of the transformation from $\eta(t)$ to $x(t)$, for which we will not require an explicit form.

The method for finding $S[x]$ is discussed in some detail in Ref. [13], but we can obtain it relatively quickly from Eqs.

(3) and (6) by first writing

$$
\begin{aligned}
{[\dot{x}+} & \left.V^{\prime}(x)\right]+\frac{2 \Gamma}{\omega_{0}^{2}}\left[\ddot{x}+\dot{x} V^{\prime \prime}(x)\right] \\
& +\frac{1}{\omega_{0}^{2}}\left[\ddot{x}+\ddot{x} V^{\prime \prime}(x)+\dot{x}^{2} V^{\prime \prime \prime}(x)\right] \\
= & \omega_{0}^{-2} \eta(t) .
\end{aligned}
$$

Since the noise $\eta$ is Gaussian, white, with strength $D$, and has zero mean, the probability functional $P[\eta]$ has the form $\exp -(1 / 4 D) \int d t \eta^{2}(t)$. A naive substitution of Eq. (9) into this expression is sufficient to give the correct functional form for $\mathcal{P}[x]$ to leading order in $D$, namely,

$$
\mathcal{P}[x]=J[x] \exp -\mathcal{A}[x] / \Delta,
$$

where

$$
\mathcal{A}[x]=S[x] / \omega_{0}^{4}, \quad \Delta=D / \omega_{0}^{4}
$$

and

$$
\begin{aligned}
\mathcal{A}[x]= & \frac{1}{4} \int_{-\infty}^{\infty} d t\left\{\left[\dot{x}+V^{\prime}(x)\right]\right. \\
& \left.+\frac{2 \Gamma}{\omega_{0}^{2}}\left[\ddot{x}+\dot{x} V^{\prime \prime}(x)\right] \frac{1}{\omega_{0}^{2}}\left[\ddot{x}+\ddot{x} V^{\prime \prime}(x)+\dot{x}^{2} V^{\prime \prime \prime}(x)\right]\right\}^{2} .
\end{aligned}
$$

Having discussed the reformulation of the problem expressed as the Langevin equation (3) as a Fokker-Planck equation or as a path integral, we are now in a position to discuss the $D \rightarrow 0$ asymptotics. In the case of the Fokker-Planck equation one may perform a WKB-like analysis, while in the case of the path-integral one may evaluate Eq. (7) by steepest descents, the paths which dominate the integral being those for which

$$
\frac{\delta \mathcal{A}[x]}{\delta x}=0 .
$$

From Eq. (12) it can be seen that a sixth-order nonlinear differential equation is obtained. The solutions of this equation, subject to the appropriate boundary conditions are the instantons or optimal paths $x_{c}(t)$ of the model. Substituting this solution back into the action gives a number $S \equiv S\left[x_{c}\right]$ $=\omega_{0}^{4} \mathcal{A}\left[x_{c}\right]$. In either case, the WKB treatment or the steepest descent evaluation of the path integral, an analysis of the conditional probability (7) leads to a rate of escape from one potential well to another which has the characteristic form $\mathcal{N} \exp -S / D$, where $\mathcal{N}$ will be termed the prefactor. In the ratchet we are interested in the current $j$ which is proportional to the difference between the rates of escape from a particular potential well to the neighboring wells on the right and on the left. It is therefore reasonable that it should have the form [2]

$$
j=\lambda\left[\mathcal{N}_{+} \exp \left(-S_{+} / D\right)-\mathcal{N}_{-} \exp \left(-S_{-} / D\right)\right]
$$

where the plus and minus symbols denote right and leftward transitions respectively and $\lambda$ is the well spacing.

In the next section we will calculate the actions in Eq. (14), by solving the sixth order equation obtained from Eq. (13), numerically. However, in order to get some intuition for what may happen we will end this section by assuming that $\omega_{0}$ is large (compared to the scale set by the curvature of the potential at the bottom of the wells) and obtaining the action as a power series in $\omega_{0}^{-2}$. In order to do this, we first rewrite the noise correlator (5) in the generic form

$$
C^{-1}(\omega)=\omega_{0}^{4}\left[1+\kappa_{1} \tau^{2} \omega^{2}+\kappa_{2} \tau^{4} \omega^{4}\right],
$$

where

$$
\tau=\omega_{0}^{-1}, \quad \kappa_{1}=-2\left(1-\frac{2 \Gamma^{2}}{\omega_{0}^{2}}\right), \quad \kappa_{2}=1
$$

With the form (15), the action for a path, starting at the bottom of a well at $x=a$ and ending at the top of an adjacent barrier at $x=b$, is given by [14] 
TABLE I. Numerical values of the integrals in Eq. (17) for the potential (2).

\begin{tabular}{ccccc}
\hline \hline & $\mathrm{a}^{(1)}$ & $\mathrm{a}^{(2)}$ & $\mathrm{a}^{(3)}$ & $\mathrm{a}^{(4)}$ \\
\hline+ & 4.62 & 22.58 & 681.41 & 751.48 \\
- & 4.62 & 5.61 & 59.06 & 70.15 \\
\hline \hline
\end{tabular}

$$
\begin{aligned}
\mathcal{A} \equiv & \mathcal{A}\left[x_{c}\right]=\int_{a}^{b} d x V^{\prime}+\kappa_{1} \tau^{2} \int_{a}^{b} d x V^{\prime}\left(V^{\prime \prime}\right)^{2} \\
& +\kappa_{2} \tau^{4} \int_{a}^{b} d x V^{\prime}\left[\left(V^{\prime \prime}\right)^{2}+V^{\prime} V^{\prime \prime \prime}\right]^{2} \\
& -\kappa_{1}^{2} \tau^{4} \int_{a}^{b} d x\left(V^{\prime}\right)^{3}\left(V^{\prime \prime \prime}\right)^{2}+O\left(\tau^{6}\right) .
\end{aligned}
$$

The $O\left(\tau^{6}\right)$ terms are also known, they are given in Ref. [14], and are proportional to $\kappa_{1}^{3}$ and $\kappa_{1} \kappa_{2}$.

First of all, suppose that the spectrum is sharply peaked, $\omega_{0}^{2} \gg 2 \Gamma^{2}$, then both $\kappa_{1}$ and $\kappa_{2}$ have magnitudes of order one. In this case the expansion (17) is simply one in powers of $\tau^{2}=\omega_{0}^{-2}$. The $O\left(\tau^{2}\right)$ corrections are necessary, otherwise $\mathcal{A}$ simply depends on the height of the barrier $\Delta V$ $=\int_{a}^{b} d x V^{\prime}(x)$ and the asymmetry of the potential does not manifest itself. An interesting special case is when $\omega_{0}$ is large, but $\omega_{0}^{2}=2 \Gamma^{2}$. Then $\kappa_{1}$ is zero, and the second and fourth terms on the right hand side of Eq. (17) vanish, as do all of the $O\left(\tau^{6}\right)$ terms. So in this case

$$
\begin{aligned}
\mathcal{A}= & \Delta V+\tau^{4} \int_{a}^{b} d x V^{\prime}\left[\left(V^{\prime \prime}\right)^{2}+V^{\prime} V^{\prime \prime \prime}\right]^{2} \\
& +O\left(\tau^{8}\right) \quad\left[\text { for } \omega_{0}^{2}=2 \Gamma^{2}\right] .
\end{aligned}
$$

If there were no $O\left(\tau^{4}\right)$ terms, it would be the case that when $\omega_{0}^{2}=2 \Gamma^{2}$, the action only depended on the barrier height, and so if the height of the barrier was the same to the right or to the left, we should not expect any net current. Moreover, if we plot the spectrum of the noise, for the particular value of $\omega_{0}^{2}=2 \Gamma^{2}$, we can see that it is very flat: the particle effectively is feeling a white noise which gives no ratchet effect at all. Since there are $O\left(\tau^{4}\right)$ corrections in Eq. (18), this is not quite so however. In order to investigate this point in a little more detail, we have calculated the integrals in Eq. (17) [and Eq. (18)] using $V(x)$ given in Eq. (2). We find

$$
\mathcal{A}_{ \pm}=a_{ \pm}^{(1)}+a_{ \pm}^{(2)} \kappa_{1} \tau^{2}+a_{ \pm}^{(3)} \kappa_{2} \tau^{4}-a_{ \pm}^{(4)} \kappa_{1}^{2} \tau^{4}+O\left(\tau^{6}\right),
$$

where the coefficients $a_{ \pm}^{(i)}$ are given in Table I.

Let us focus on the particular values $\omega_{0}=10.5$ and $\omega_{0}$ $=31.6$ which we will use later. A short calculation using Eq. (19) and the values of $a_{ \pm}^{(i)}$ given in Table I above shows, that to $O\left(\tau^{6}\right), \mathcal{A}_{+}=\mathcal{A}_{-}$when $\Gamma=6.84$ (for $\omega_{0}=10.5$ ) and $\Gamma$ $=22.13$ (for $\omega_{0}=31.6$ ). If we had used the result $\omega_{0}^{2}=2 \Gamma^{2}$ — valid for small $\tau$ as indicated by Eq. (18) — the corresponding values of $\Gamma$ would have been 7.42 and 22.34 , re- spectively. So we see that $\Gamma=\omega_{0} / \sqrt{2}$ is a reasonable estimate for the value at which $\mathcal{A}_{+}$and $\mathcal{A}_{-}$become equal when $\omega_{0}$ has the larger value (31.6), but it is considerably different in the case when $\omega_{0}$ is smaller: $\omega_{0}=10.5$. We shall discuss the interpretation of the point where the actions for the leftand right-moving transitions become equal in more detail in the concluding section.

\section{CALCULATION OF LEADING CONTRIBUTION}

In this section we calculate the leading small $D$ contributions $S_{ \pm}$(or alternatively the leading small $\Delta$ contributions $\mathcal{A}_{ \pm}$) to the current $j$ in Eq. (7) for the case of the potential (2). In the preceding section we illustrated the general idea by evaluating these actions for large $\omega_{0}$, but a general analytic treatment is not possible and we will have to resort to a numerical calculation of their values. From Eq. (12), the general form of the action functional is

$$
\mathcal{A}[x]=\int_{t_{0}}^{t} d t L(\dot{x}, \ddot{x}, \cdots ; x) .
$$

The variation (13) leads to an Euler-Lagrange equation of sixth order

$$
\sum_{j=0}^{3}(-1)^{j} \frac{d^{j}}{d t^{j}}\left(\frac{\partial L}{\partial x^{(j)}}\right)=0
$$

where $x^{(j)} \equiv d^{j} x / d t^{j}$. A numerical solution will involve the decomposition of this equation into six first-order differential equations. A systematic procedure for achieving this is provided by the Hamiltonian formulation for the generalized mechanics given by Eqs. (20) and (21) [15].

If we carry out this procedure starting from the Lagrangian given by

$$
\begin{aligned}
L(x, \dot{x}, \ddot{x}, \ddot{x})= & \frac{1}{4}\left\{\left[\dot{x}+V^{\prime}(x)\right]+\frac{2 \Gamma}{\omega_{0}^{2}}\left[\ddot{x}+\dot{x} V^{\prime \prime}(x)\right]\right. \\
& \left.+\frac{1}{\omega_{0}^{2}}\left[\ddot{x}+\ddot{x} V^{\prime \prime}(x)+\dot{x}^{2} V^{\prime \prime \prime}(x)\right]\right\}^{2},
\end{aligned}
$$

we find the following Hamiltonian:

$$
\begin{aligned}
H(\vec{x}, \vec{p})= & p_{1} x_{2}+p_{2} x_{3}+\omega_{0}^{4} p_{3}^{2}-p_{3}\left\{\omega_{0}^{2}\left(x_{2}+V^{\prime}\right)+2 \Gamma\left(x_{3}\right.\right. \\
& \left.\left.+x_{2} V^{\prime \prime}\right)+x_{3} V^{\prime \prime}+x_{2}^{2} V^{\prime \prime \prime}\right\},
\end{aligned}
$$

where $\vec{x}=\left(x_{1}, x_{2}, x_{3}\right)$ and $\vec{p}=\left(p_{1}, p_{2}, p_{3}\right)$. The action turns out to be [15]

$$
\mathcal{A}=\int_{-\infty}^{\infty} p_{3}^{2} d t
$$

Hamilton's equations have their usual form:

$$
\dot{x}_{i}=\frac{\partial H}{\partial p_{i}}, \quad \dot{p}_{i}=-\frac{\partial H}{\partial x_{i}}, \quad i=1, \ldots, 3
$$


they are, by construction, equivalent to the Euler-Lagrange equations (21). Using the Hamiltonian (23), the six equations (25) yield

$$
\begin{gathered}
\dot{x}_{1}=x_{2}, \\
\dot{x}_{2}=x_{3}, \\
\dot{x}_{3}=2 \omega_{0}^{4} p_{3}-\left\{\omega_{0}^{2}\left[x_{2}+V^{\prime}\left(x_{1}\right)\right]+2 \Gamma\left[x_{3}+x_{2} V^{\prime \prime}\left(x_{1}\right)\right]\right. \\
\left.+x_{3} V^{\prime \prime}\left(x_{1}\right)+x_{2}^{2} V^{\prime \prime \prime}\left(x_{1}\right)\right\}, \\
\dot{p}_{1}=p_{3}\left\{\omega_{0}^{2} V^{\prime \prime}\left(x_{1}\right)+2 \Gamma x_{2} V^{\prime \prime \prime}\left(x_{1}\right)+x_{3} V^{\prime \prime \prime}\left(x_{1}\right)+x_{2}^{2} V^{\prime \prime \prime \prime}\left(x_{1}\right)\right\}, \\
\dot{p}_{2}=-p_{1}+p_{3}\left\{\omega_{0}^{2}+2 \Gamma V^{\prime \prime}\left(x_{1}\right)+2 x_{2} V^{\prime \prime \prime}\left(x_{1}\right)\right\}, \\
\dot{p}_{3}=-p_{2}+p_{3}\left\{2 \Gamma+V^{\prime \prime}\left(x_{1}\right)\right\} .
\end{gathered}
$$

For an escape problem, we are searching for solutions that provide the minimum of the action. Imposing the condition that the variation with time of the action is zero, as before, $H=0$, and following previous work [15], we choose as boundary conditions for the "uphill" solution (going from the bottom to the top of the potential)

$$
\begin{gathered}
x_{1}(-\infty)=x_{\min }, x_{1}(\infty)=x_{\max }, \\
x_{2}( \pm \infty)=0, \\
x_{3}( \pm \infty)=0 .
\end{gathered}
$$

In order to solve Eqs. (26) -(31) in practice we have to truncate them to a large, but finite, time interval and use the boundary conditions

$$
\begin{gathered}
x_{1}(-T)=x_{\text {min }}, x_{1}(0)=x_{\max }, \\
x_{2}(-T)=0, x_{2}(0)=0, \\
x_{3}(-T)=0, x_{3}(0)=0,
\end{gathered}
$$

where we have used the time translation invariance of the equations.

The TWPBVP subroutines developed for solving two-point boundary value problem were used [16]. In order to get convergence we used the following procedures. We linearized Eqs. (26)-(31) at the initial point, and changed the boundary conditions at that point, perturbing them in the direction of the unstable manifold given by eigenvectors with eigenvalues having positive real part. We took as an initial guess a straight line joining the boundary points, and the solution of this modified problem was used as initial guess to the original problem, leading to a solution for the optimal path.

Having found this solution, the action

$$
\mathcal{A}=\int_{-T}^{0} p_{3}^{2} d t
$$

was calculated, and to minimize the effect of the cutoff effect we added the correction from integrating the linear expres- sions on the boundaries from $-\infty$ to $-T$ and from 0 to $\infty$. When $T$ is chosen to be large enough, the result is independent of its value.

The results of this calculation will be discussed in Sec. VI where they will be compared to the simulations that we have also carried out on this problem. We now turn to a discussion of these simulations.

\section{ANALOG AND NUMERICAL SIMULATIONS}

In this section we study our correlation ratchet using ana$\log$ and digital simulations. We have measured the variation of the current with $\Gamma$ in two ways: analog simulation experiments on an electronic circuit and Monte-Carlo simulations on a digital computer.

The first of these techniques [17-19] involves the building of an electronic circuit to model the system under study, the application of appropriate forces, and analysis of the response by means of a digital computer. The absence of truncation errors makes analog simulations especially valuable for use, e.g., with fast oscillating systems where the integration time (the time over which data are accumulated and perhaps ensemble-averaged) substantially exceeds the vibration period, as occurs with QMN. Digital techniques have the advantage that they can always in principle be made more accurate than analog methods, which typically achieve $2-3 \%$ accuracy, but the relative simplicity of analog simulations and their realism (being much closer to a real experiment than a digital simulation) represent significant advantages.

The electronic circuit used to model (3) and (6) is shown as a block diagram in Fig. 4. The lower section is the harmonic oscillator used as a "filter" to convert [8] quasiwhite noise from a feedback shift-register noise generator [20,19] into QMN. The QMN is then applied to the input of the upper part of the circuit, which models the ratchet potential itself. Although the basis of the circuit is standard [19], several points of detail deserve amplification. The force corresponding to the trigonometric potential of Eq. (2) is created using trigonometric identities to write it as

$$
V^{\prime}(x)=-\frac{29}{10} \sin x+\cos x-\frac{4}{3} \sin x \cos x+\frac{6}{5} \sin ^{3} x,
$$

so we can build the force using only two AD639 ICs [21]. An inherent limitation of the AD639 IC is that it can only treat a restricted range of angles $\left( \pm 500^{\circ}\right)$. To prevent its input from straying outside this range, provision is made for resetting the circuit using DG303AC [22] switches (they have not been plotted in the block diagram) [17-19]. The voltage in the circuit corresponding to coordinate $x$ was digitized with a 12-bit Microstar ADC [23], model DAP 3200a/ 415. Data analysis exploited the on-board $100 \mathrm{MHz}$ Intel 486DX coprocessor, which was operated within a MatLabbased PC software system developed by Kaufman [24].

The digital simulations were done using a specialized algorithm, described in Ref. [8], which we will briefly recall here. The particular structure of Eqs. (3) and (6) poses, in principle, a problem if a simple minded algorithm is used in the integration: Eq. (6) is characterized by two time scales $\left(\omega_{0}\right.$ and $\left.\Gamma\right)$ and the integration time step (call it $\left.h\right)$ used in the 


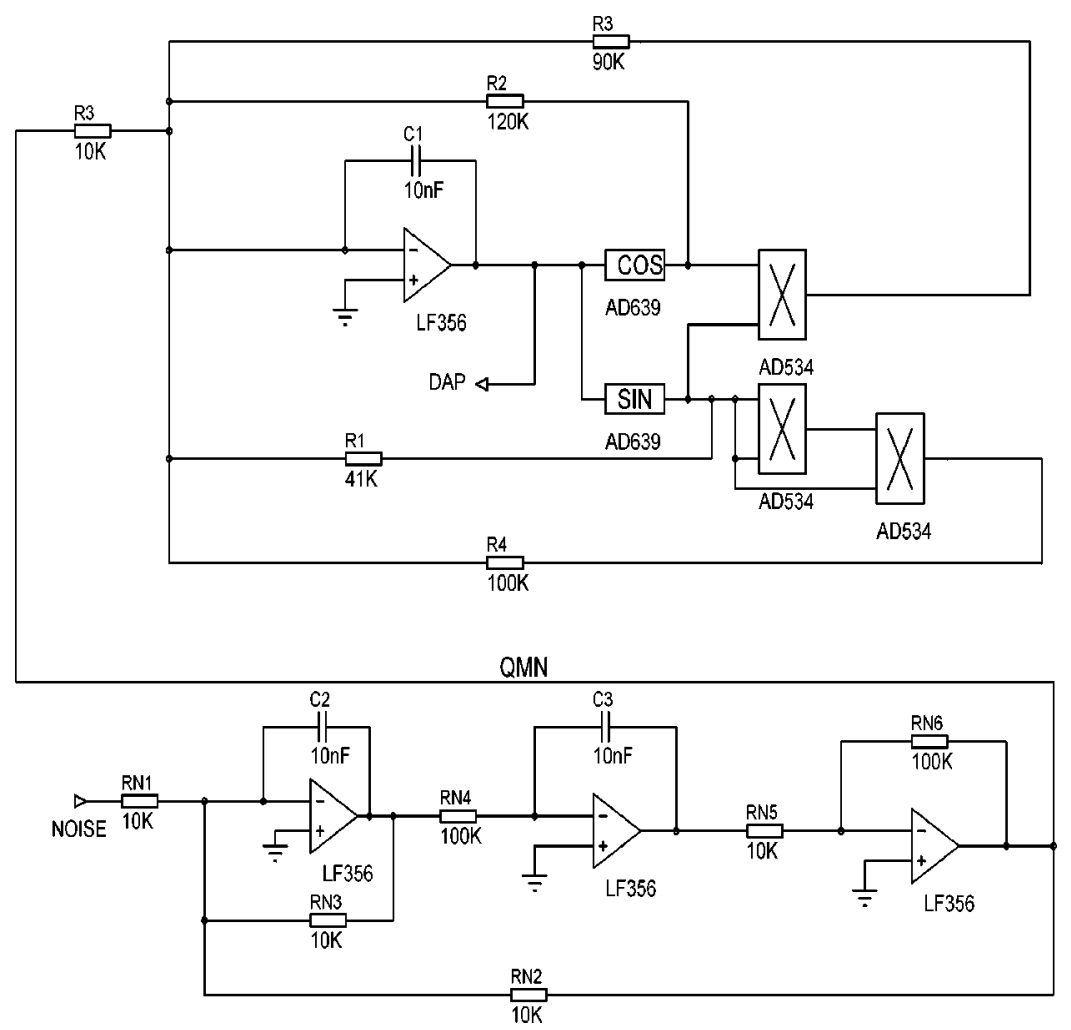

FIG. 4. Experimental analog circuit.

digital simulations would be chosen in such a way that both $\omega_{0} h \ll 1$ and $\Gamma h \ll 1$. Also, if $\tau_{r}$ is the typical relaxation time in Eq. (3), we should also satisfy $h / \tau_{r} \ll 1$. This latter inequality, for the typical parameters which are of physical interest, is normally satisfied as soon as the former one is: in other words, in a simple minded algorithm the constraint on the integration time step comes from Eq. (6) rather than from Eq. (3), because the time scales involved by $\omega_{0}$ and $\Gamma$ are (much) smaller than $\tau_{r}$. From the point of view of the problem we are trying to solve, however, this would not be very efficient: we would be using most of the CPU time integrating the noise equation (6) rather than integrating the dynamical equation representing the model under study.

The particular structure of Eqs. (3) and (6) suggests that a specialized algorithm could be more efficient: the point is that Eq. (6) is a linear filtering of an uncorrelated Gaussian noise. This means that the output of this equation (the variable $\xi$ ) is itself a Gaussian variable, of unknown intensity and correlation: hence, it may be possible to integrate Eq. (3) directly, working out the appropriate integration algorithm, using the statistical properties of the Gaussian variable $\xi$. The algorithm used to integrate Eq. (3) is the Heun algorithm [25] which prescribes that we integrate Eq. (3) with a couple of elementary steps, namely we first predict

$$
\tilde{x}(h)=x(0)+h\left[-V^{\prime}(x(0))\right]+r(h),
$$

and then correct as

$$
x(h)=\left\{\tilde{x}(h)+x(0)+h\left[-V^{\prime}(\tilde{x}(h))\right]+r(h)\right\} / 2 .
$$

In the expression above, we need to evaluate the quantity $r(h) \equiv \int_{0}^{h} \xi(s) d s$, which can be written [8] as

$$
r(h)=A_{31} \xi(0)+A_{32} \dot{\xi}(0)+w_{3},
$$

where

$$
w_{3}=B_{31} z_{1}+B_{32} z_{2}+B_{33} z_{3},
$$

and where $z_{i}$ are uncorrelated Gaussian deviates of average zero and standard deviation one. Note that the quantity $r(h)$ turns out to be a linear combination of Gaussian variables, as expected. Defining $\Omega^{2} \equiv \omega_{0}^{2}-\Gamma^{2}$ and $\lambda_{ \pm} \equiv-\Gamma \pm \sqrt{\Gamma^{2}-\omega_{0}^{2}}$, we have

$$
\begin{gathered}
A_{31}=\frac{i}{2 \Omega}\left\{\frac{\lambda_{-}}{\lambda_{+}}\left(e^{h \lambda_{+}}-1\right)-\frac{\lambda_{+}}{\lambda_{-}}\left(e^{h \lambda_{-}}-1\right)\right\}, \\
A_{32}=\frac{i}{2 \Omega}\left\{\frac{e^{h \lambda_{-}-1}}{\lambda_{-}}-\frac{e^{h \lambda_{+}}-1}{\lambda_{+}}\right\} .
\end{gathered}
$$

The expressions for $B_{i j}$ are very cumbersome, and we refer the reader to Ref. [8]: note that Ref. [8] contains a misprint, the quantity $4 \pi T / 2 \Omega^{2}$ on the right hand side of Eq. (A14) should read $\Gamma T / \Omega^{2}$.

A warning is in order concerning the random noise generator. The noise intensities of interest are fairly small compared to the barrier that the Brownian particle has to overcome to diffuse and generate a net current. It is then of great importance to make sure that the rare activation events are correctly generated, which implies that the noise generator should be particularly accurate in generating the tails of the distribution. The generator used works by generating a Gaussian random variable, using the Ziggurath algorithm [26], from flat random distributions obtained with a subtract and carry algorithm [27,28].

The actions $A_{ \pm}$were calculated from the slope of plotting the logarithm of the mean escape time, calculated as the total time of observation divided by the number of transitions to the left or right, versus $1 / D$. The current in the experiments is 


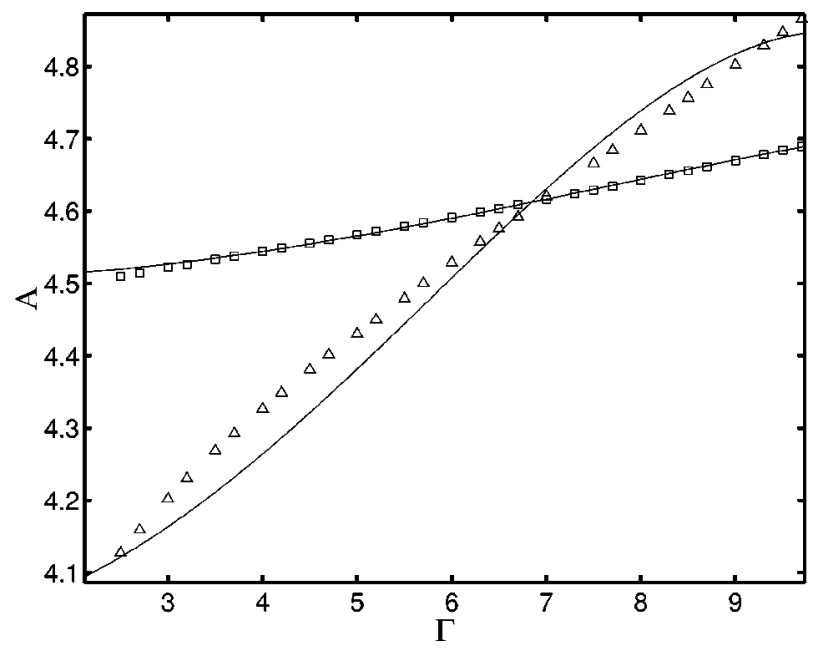

FIG. 5. Analytic (small $\tau$ ) versus numerical (general $\tau$ ) actions for $\omega_{0}=10.5$. Squares are for escapes to the left, triangles to the right. Symbols are from numerical integration, solid lines from analytic calculations.

easily obtained by keeping track of the distance moved by the random walker and dividing it by the total simulation time. These results can be observed in the figures.

\section{ANALYSIS OF RESULTS AND CONCLUSIONS}

In this section we wish to compare the theoretical predictions of Sec. III, IV with the experiments. Our main aim is to understand the structure of the current (14). This is made up of actions $S_{ \pm}$and prefactors $\mathcal{N}_{ \pm}$. For small $D$, the action dominates, so we begin by comparing the actions calculated from Eq. (17) with the numerical method discussed in Sec. IV. For a typical value of $\omega_{0}=10.5$, the results are shown in Fig. 5. This shows reasonable agreement between analytical and numerical results of solving the full set of equations of Sec. IV. This fully justifies the approximation of Sec. III, which is very useful given the difficulty of carrying out the numerical integrations in the method of Sec. IV.

Now we are in a position to compare the theoretical predictions with the digital simulation of Sec. V. This is shown in Fig. 6. The digital simulation shows the same trends as the

TABLE II. Analog $\left(j_{A}\right)$ and digital $\left(j_{D}\right)$ currents for $\omega_{0}=10.5$.

\begin{tabular}{cccc}
\hline \hline$\Gamma$ & $\Delta$ & $j_{A}$ & $j_{D}$ \\
\hline 0.938 & 0.564 & $2.17 \times 10^{-4}$ & $1.53 \times 10^{-3}$ \\
1.346 & 0.591 & $2.31 \times 10^{-4}$ & $1.26 \times 10^{-3}$ \\
2.386 & 0.617 & $2.14 \times 10^{-4}$ & $8.10 \times 10^{-4}$ \\
5.250 & 0.654 & $1.20 \times 10^{-4}$ & $1.74 \times 10^{-4}$ \\
6.402 & 0.670 & $4.32 \times 10^{-5}$ & $1.03 \times 10^{-5}$ \\
7.721 & 0.673 & $-1.29 \times 10^{-5}$ & $-1.78 \times 10^{-4}$ \\
9.375 & 0.681 & $-1.25 \times 10^{-5}$ & $-3.15 \times 10^{-4}$ \\
11.170 & 0.693 & $-3.84 \times 10^{-5}$ & $-4.64 \times 10^{-4}$ \\
13.462 & 0.713 & $-1.20 \times 10^{-4}$ & $-6.54 \times 10^{-4}$ \\
17.500 & 0.737 & $-2.13 \times 10^{-4}$ & $-7.78 \times 10^{-4}$ \\
21.000 & 0.796 & $-3.60 \times 10^{-4}$ & $-1.12 \times 10^{-3}$ \\
26.250 & 0.832 & $-4.33 \times 10^{-4}$ & $-1.28 \times 10^{-3}$ \\
29.167 & 0.863 & $-5.44 \times 10^{-4}$ & $-1.42 \times 10^{-3}$ \\
\hline \hline
\end{tabular}

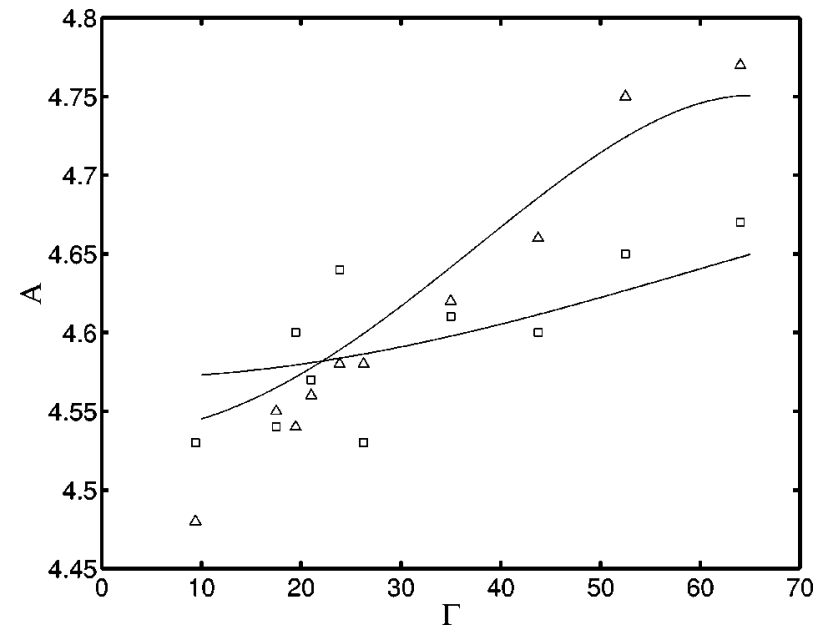

FIG. 6. Digital simulations and theoretical actions, for $\omega_{0}$ $=31.6$. Squares denotes escapes to the left, triangles to the right. Symbols are simulations, curves theory.

analytical results, but there is a large amount of scatter. Nevertheless, the value of $\Gamma$ at the point where $\mathcal{A}_{+}=\mathcal{A}_{-}$predicted by the digital simulations is in reasonable agreement with the theoretical value.

The prefactors are, unfortunately, difficult to calculate. In fact, a calculation for QMN has not yet been carried out. The prefactors are, however, known for white noise and exponentially correlated noise (for small noise correlation time [29]), and we use these in the expectation that they are a reasonable approximation to the true result. In Fig. 7, the current calculated from Eq. (14) using these prefactors together with an action calculated as in Sec. IV, is plotted with the data from the digital simulation. A possible interpretation of the deviation of the theoretical from the experimental results could be the approximate prefactor. However, it is clear that the exponentially correlated prefactor is an improvement over the white noise one, and this suggests that the correct QMN prefactor might give even better agreement. In any case, it is clearly demonstrated that a ratchet consisting of an asymmetric periodic potential plus quasimonochromatic noise forcing can indeed give rise to a net transport of particles.

Table II displays the current obtained for $\omega_{0}=10.5$ in both the analog experiment and the digital simulation. In this

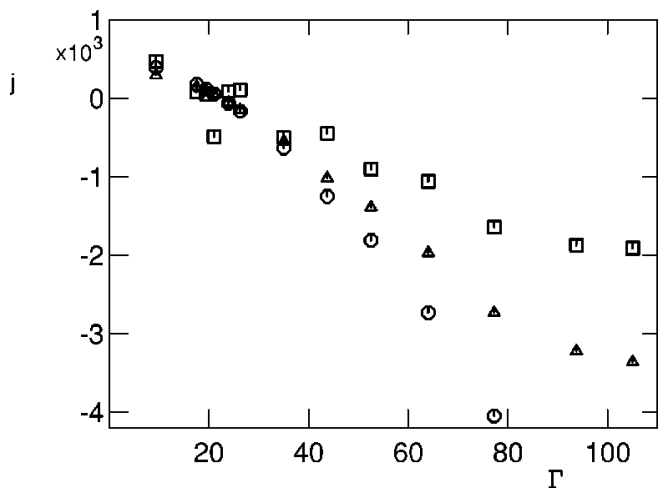

FIG. 7. Current for $\omega_{0}=31.6$ and $\Delta=D / \omega_{0}^{4}=0.87$. The squares are from digital simulation; the circles represent theory for a white noise prefactor; the triangles represent theory with the improved prefactor. 
case, although actions can be calculated analytically, the parameter in the expansion for the prefactor is no longer small and one is unable to obtain an analytic expression for the current. The values $\omega_{0}=10.5$ and $\omega_{0}=31.6$ were chosen for technical reasons connected with carrying out the analog experiment.

There is no reason to assume that the current reversal $(j$ $=0$ ) necessarily occurs when $\mathcal{A}_{+}=\mathcal{A}_{-}$, because the prefactors may cause some deviation from this leading order result. However, from Fig. 7 and Table II it seems that they do in fact occur at the same point — even though the magnitude of the analog current is consistently less than that of the digital current. This may be because, as $D \rightarrow 0$, the action completely dominates or because the prefactors happen to be approximately equal at this point.

In the literature, this problem has been already discussed in Refs. [2] and [30]. In Ref. [2] it was found that there is indeed a net current in the system, and, working in the limit of small $\Gamma / \omega_{0}$, the authors were able to show that the sign of the current changes as the curvature at $\omega=0$ [i.e., $\left.C^{\prime \prime}(0)\right]$ changes with varying $\Gamma$. In Ref. [30] the authors considered a model of the form (3), but with an additional white noise. However, as we have seen, there is no need to introduce an additional noise of this type in order to see a current reversal.
The authors found that the current should change sign for at least two different values of $\Gamma$.

Our aim has been to study how the current changed as the noise parameters $\Gamma$ and $\omega_{0}$ varied, for finite $\Gamma / \omega_{0}$. We found that that the change in the spectral density curvature at $\omega$ $=0$ mentioned above is still the main effect in determining the current direction, in agreement with Ref. [2]. We have included in the theoretical treatment higher order terms in $\Gamma / \omega_{0}$ : our result coincides with the result of Ref. [2] in the appropriate limit, with a small shift in the transition point if $\Gamma / \omega_{0}$ is finite. The simulations which were carried out support the theoretical conclusions. We have not observed more than one current reversal experimentally (with fixed $\omega_{0}$ and varying $\Gamma$ ), but examination of Eq. (19) shows that there is another solution [to $O\left(\tau^{4}\right)$ ] for which $\mathcal{A}_{+}=\mathcal{A}_{-}$. It would be interesting to explore this regime in more detail experimentally.

\section{ACKNOWLEDGMENTS}

We are grateful to M. I. Dykman for valuable discussions and encouragement during the early stages of the project. This work was supported in part by the Engineering and Physical Sciences Research Council under Grant No. GR/ K49966, and by INTAS under Grant No. 97-574.
[1] M. O. Magnasco, Phys. Rev. Lett. 71, 1477 (1993).

[2] M. M. Millonas and M. I. Dykman, Phys. Lett. A 183, 65 (1994).

[3] C. Doering, W. Horsthemke, and J. Riordan, Phys. Rev. Lett. 72, 2984 (1994).

[4] Ya. M. Blanter and M. Büttiker, Phys. Rev. Lett. 81, 4040 (1998).

[5] For reviews see, for example, F. Jülicher, A. Ajdari, and J. Prost, Rev. Mod. Phys. 69, 1269 (1997); M. Bier, Contemp. Phys. 38, 371 (1997).

[6] R. P. Feynman, R. B. Leighton, and M. Sands, The Feynman Lectures on Physics (Addison-Wesley, Reading, MA, 1966), Vol. I, Chap. 46.

[7] M. Bier and R. D. Astumian, Phys. Rev. Lett. 76, 4277 (1996).

[8] M. I. Dykman, R. Mannella, P. V. E. McClintock, N. D. Stein, and N. G. Stocks, Phys. Rev. E 47, 3996 (1993).

[9] M. Arrayás, R. Mannella, P. V. E. McClintock, A. J. McKane, and N. D. Stein, in Noise in Physical Systems and 1/f Fluctuations, edited by C. Claeys and E. Simoen (World Scientific, Singapore, 1997).

[10] A. Igarashi and T. Munakata, J. Phys. Soc. Jpn. 57, 2439 (1988).

[11] L. Schimansky-Geier and Ch. Zülicke, Z. Phys. B 79, 451 (1990).

[12] A. J. McKane, H. C. Luckock, and A. J. Bray, Phys. Rev. A 41, 644 (1990), Sec. V.

[13] S. J. B. Einchcomb and A. J. McKane, in Fluctuations and Order, edited by M. Millonas (Springer-Verlag, New York, 1996).

[14] A. J. McKane, Phys. Rev. A 40, 4050 (1989).

[15] S. J. B. Einchcomb and A. J. McKane, Phys. Rev. E 51, 2974 (1995).

[16] J. R. Cash and M. H. Wright, SIAM (Soc. Ind. Appl. Math.) J.
Sci. Stat. Comput. 12, 971 (1991).

[17] L. Fronzoni, in Noise in Nonlinear Dynamical Systems, edited by F. Moss and P. V. E. McClintock (Cambridge University Press, Cambridge, 1989), Vol. 3, pp. 222-242.

[18] P. V. E. McClintock and F. Moss, in Noise in Nonlinear Dynamical Systems (Ref. [17]), Vol. 3, pp. 243-274.

[19] D. G. Luchinsky, P. V. E. McClintock, and M. I. Dykman, Rep. Prog. Phys. 61, 889 (1998).

[20] S. Faetti, C. Festa, L. Fronzoni, P. Grigolini, and P. Martano, Phys. Rev. A 30, 3252 (1984).

[21] The AD639BD integrated cicuit is from Analog Devices, Box 9106, Norwood, MA 02062.

[22] The DG303AC integrated cicuit is from Siliconix, 2201 Laurelwood Road, Santa Clara, CA 95054.

[23] Microstar Laboratories Inc., 2265 116th Avenue NE, Bellevue, WA 98004; see URL www.mstarlabs.com. The UK distributor is Amplicon Liveline Ltd, Centenary Industrial Estate, Hollindean Road, Brighton BN2 4AW.

[24] I. Kh. Kaufman, M. Arrayás, D. G. Luchinsky, and P. V. E. McClintock (unpublished).

[25] A. Greiner, W. Strittmatter, and J. Honerkamp, J. Stat. Phys. 51, 95 (1988); see also R. Mannella, in Supercomputation in Nonlinear and Disordered Systems: Algorithms, Applications and Architectures, edited by F. L. Vasquez, F. Tirando, and I. Martin (World Scientific, Singapore, 1997), pp. 100-130.

[26] G. Marsaglia and W. W. Tsang, SIAM (Soc. Ind. Appl. Math.) J. Sci. Stat. Comput. 5, 349 (1984).

[27] M. Luscher, Comput. Phys. Commun. 79, 100 (1994).

[28] F. James, Comput. Phys. Commun. 79, 111 (1994).

[29] H. C. Luckock and A. J. McKane, Phys. Rev. A 42, 1982 (1990).

[30] R. Bartussek, P. Hänggi, B. Lindner, and L. SchimanskyGeier, Physica D 109, 17 (1997). 